\title{
Emmy Noether - „das Experiment, eine Frau zum Ordinarius zu machen“
}

\author{
Reinhard Siegmund-Schultze
}

\begin{abstract}
Der Artikel legt dar, mit welchen Argumenten Emmy Noethers Kollegen Adolf Fraenkel und Helmut Hasse 1928 ihre mögliche Berufung nach Kiel ausschlossen. Neben teilweise gerechtfertigten aber scheinheiligen Zweifeln an Noethers Eignung für elementarmathematische Vorlesungen waren es anscheinend selbstbezogene Minderwertigkeitsgefühle auf Fraenkels Seite und generelle Vorurteile gegen Frauen im akademischen Bereich bei Hasse.
\end{abstract}

Es ist Oktober 1928 und Ernst Steinitz (1871-1928), der Begründer der modernen abstrakten Körpertheorie, ist gerade in Kiel verstorben. Sein Kollege Adolf (Abraham Halevi) Fraenkel (1891-1965) bereitet an der Kieler Universität die Fakultätsliste für die Nachfolge vor.

Emmy Noether (1882-1935), die Begründerin der modernen strukturellen Algebra, die in Göttingen nur eine außerordentliche Professur innehatte, im Grunde eine Privatdozentur mit kümmerlichem Lehrauftrag, war mathematisch eine starke Kandidatin für Steinitz' Nachfolge. Ihre Arbeiten standen deutlich erkennbar in seiner Tradition. In diesem Beitrag, der sich auf unveröffentlichtes Archivmaterial stützt, ${ }^{1}$ soll es darum gehen, herauszufinden, warum Noether zwar kurz von Fraenkel als Nachfolgerin erwo- gen und auch im Fakultätsvorschlag in der Präambel erwähnt wurde, aber letzten Endes keine Chance auf eine Berufung nach Kiel hatte. Genauer gesagt geht es darum, warum Fraenkel und Helmut Hasse (1898-1979), der erste überzeugt von der Ungerechtigkeit ihrer bisherigen Zurücksetzung, der zweite Noethers enger Forschungspartner und Koautor, ${ }^{2}$ sich nicht aktiv für sie einsetzten.

Der mit ihm befreundete Helmut Hasse, Entdecker des Lokal-Global-Prinzips in der Zahlentheorie, war von Fraenkel in das Berufungsverfahren einbezogen worden. Hasse, damals ordentlicher Professor in Halle, war Schüler Kurt Hensels (1861-1941) in Marburg, wie auch Fraenkel selbst.

Nach übereinstimmendem Urteil von Mathematikern und Historikern gehören Noether und Hasse zu den interna-
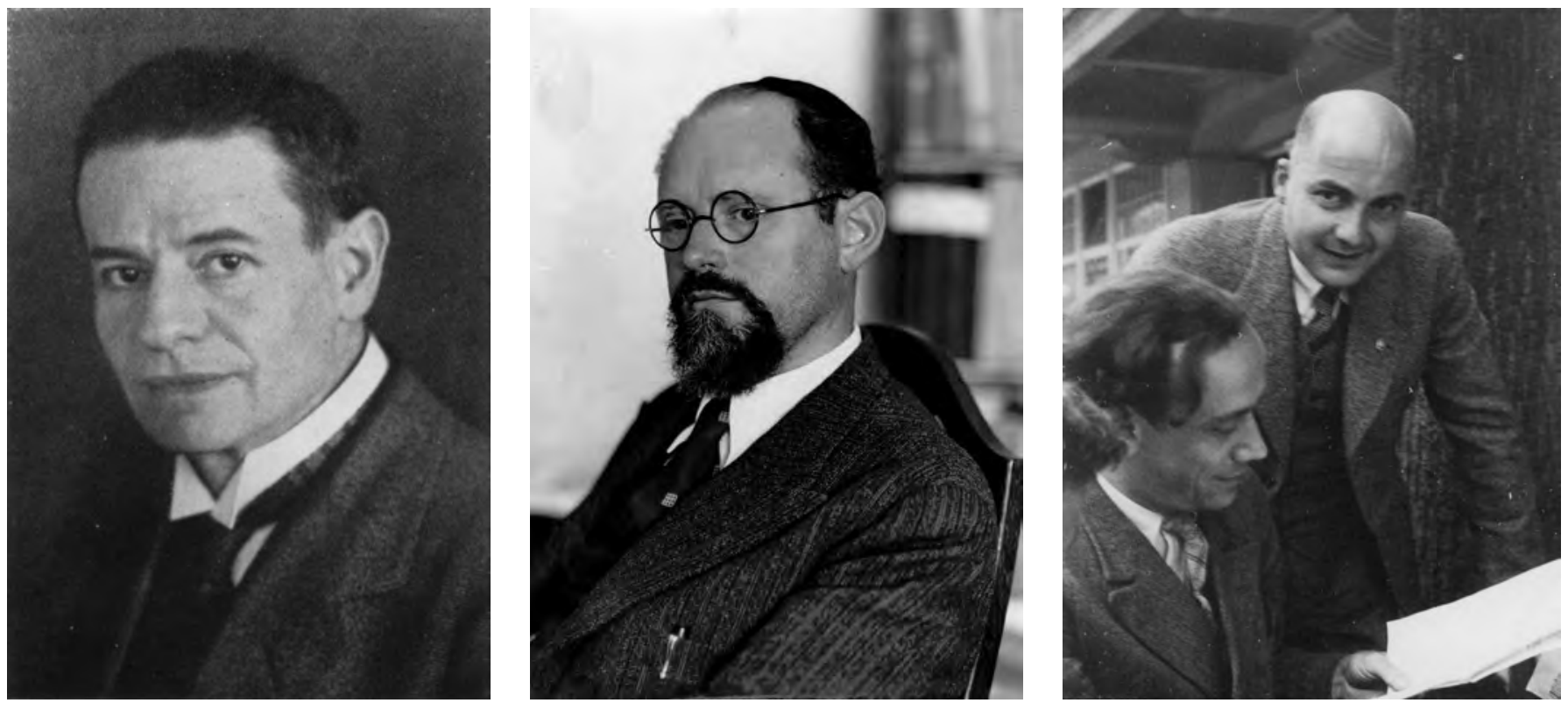

Links: Ernst Steinitz (Bildarchiv des Mathematischen Forschungsinstituts Oberwolfach/Archiv von Peter Roquette)

Mitte: Adolf Fraenkel (The David B. Keidan Collection of Digital Images from the Central Zionist Archives)

Rechts: Helmut Hasse (stehend) und Emil Artin (Bildarchiv des Mathematischen Forschungsinstituts Oberwolfach/Archiv von Peter Roquette) 


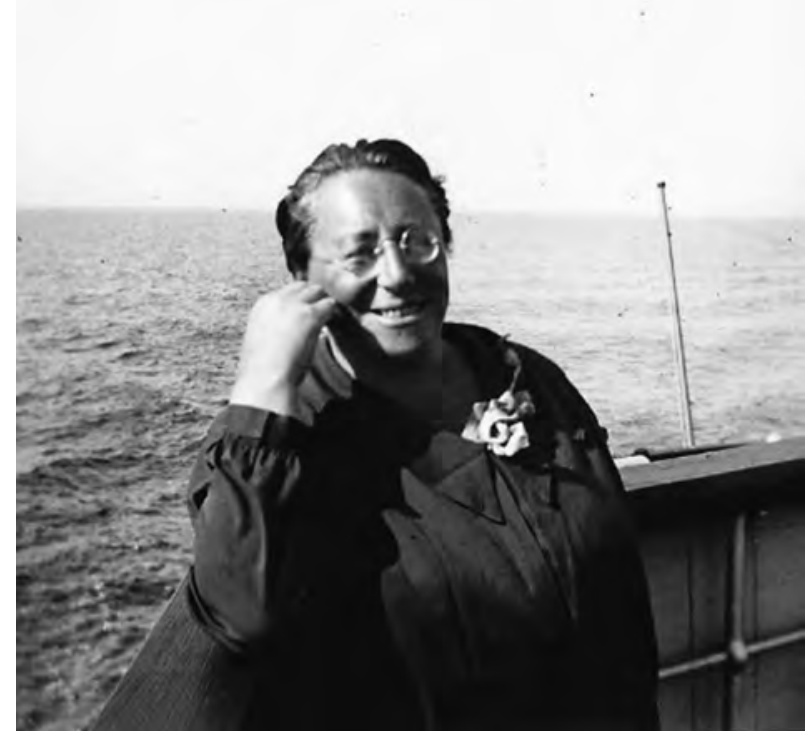

Emmy Noether, aufgenommen im September 1930 von Helmut Hasse, auf der gemeinsamen Fahrt über die Ostsee zur Tagung der DMV in Königsberg (Archiv von Peter Roquette)

tional bedeutendsten Mathematikern des 20. Jahrhunderts. Beide wurden zu Plenarvorträgen auf den vierjährlichen internationalen Mathematikerkongressen (ICM) eingeladen; Noether 1932 in Zürich, Hasse 1936 in Oslo. Fraenkel war wegen seiner Arbeiten in der Mengenlehre, insbesondere durch das Zermelo-Fraenkelsche Axiomensystem, sicherlich nicht weniger bekannt als Noether und Hasse, aber doch eindeutig nicht so vielseitig und einflussreich, unter anderem was die Anzahl und die Bedeutung seiner Schüler betrifft.

Fraenkels und Hasses politische und menschliche Haltungen „wir sind in 1. Linie Menschen, nur in 2. Mathematiker"

Um die Haltungen von Fraenkel und Hasse zu politischen Fragen und damit auch zur Frauenfrage besser verstehen zu können, sind einige Bemerkungen erforderlich. Was Fraenkel betrifft, stützen sie sich zum Teil auf Fraenkels Autobiografie „Lebenskreise“ von 1967,3 zum Teil auf seine Korrespondenz mit Hasse. Aus orthodox jüdischem Hause stammend, hatte sich Fraenkel besonders unter dem Eindruck der Katastrophe des Weltkrieges immer mehr zionistischem Gedankengut geöffnet. Nur in der Gründung einer jüdischen Heimstatt sah Fraenkel Abhilfe für das Leid vieler jüdischer Mitbürger in Europa, die oft nicht einmal volle Bürgerrechte besaßen.

Fraenkels soziales Mitgefühl mit Unterprivilegierten, das ihn von manchen seiner Professorenkollegen in der Weimarer Republik unterschied, wird auch an einigen Stellen seines Briefwechsels mit Hasse deutlich. Als er in der Zeit von Massenarbeitslosigkeit, Notverordnungen und Gehaltskürzungen, auch für Professoren, zur Geburtstagsfeier seines Lehrers Hensel nach Marburg eingeladen wurde,

\section{Die Struktur der R. Brauerschen Algebrenklassengruppe} über einem algebraischen Zahlkörper.

Insbesondere Begründung der Theorie des Normenrestsymbols und Herleitung des Rexiprozitätsgesetzes mit nichtkommutativen Hilfsmitteln.

Emmy Noether sum 50. Geburtstag am 23. Mărz 1932.

Von

Helmut Hasse in Marburg.

Einleitung.

Emmy Noether [4] hat wohl zuerst den Gedanken auggesprochen, die Theorie der nichtkommutativen Algebren sei von einfacheren Gesetzmäßigkeiten beherrscht als die Theorie der kommutativen AJgebren, insbesondere der kommutativen aigebraischen Frweiterungskörper, und folgerichtig sei die nichtkommutative Theorie in einem systematischen Aufbau nicht nur rein aีnßerlich der kommutativen Theorie voranzustellen, sondern auch zu deren Begründung sachlich weitgehend heranzaziehen. Sie hat selbst die Durchführbarkeit dieses Gedankens für verschiedene Finzelabschnitte der Gesamttheorie dargetan, und zwar nicht nur für rein-algebraische Teile (Galoissche Theorie), sondern neuerdings auch für tiefliegende arithmetische Gedankenreihen (Hauptgeschlechtssatz).

Hasses Einleitung des Artikels [3] zu Noethers 50. Geburtstag

schrieb der mehrfache Vater am 13. Dezember 1931 an Hasse:

Es reicht einfach nicht. Das Gehalt ist niedriger als bei der Ankunft hier. Ich sage das nur zur Begründung meines Nichtkommens, denn im Grunde haben wir es ja immer noch viel besser als die meisten im Volke. ${ }^{4}$

An verschiedenen Stellen des Briefwechsels wird deutlich, dass Fraenkel extrem nationalistische Gefühle, wie sie viele deutsche Professoren nach der Niederlage im Krieg und den Bestimmungen des Versailler Vertrages hegten, nicht teilte. Der Nazi-Fragebogen, den Fraenkel vor seiner Entlassung 1933 in Kiel ausfüllen musste, enthält die Information, dass er zeitweise dem „republikanischen Lehrerbund“ angehörte. 5

Im Zusammenhang mit der sogenannten Bologna-Affäre um die Teilnahme am ICM in Bologna 1928 schrieb Fraenkel an Hasse am 14. Juni 1928:

Betr. Bologna bin ich nicht ganz Ihrer Meinung, obgleich deren eindrucksvolle Äußerung mich sehr bewegt $\mathrm{u}$. auch praktisch beeinflußt hat und mich zu aufrichtigem Dank verpflichtet. Ich hatte ursprünglich genau Ihre Meinung. [...] Inzwischen aber hat auf mich [...] ein gewohnt glänzendes Plaidoyer Hilberts Eindruck gemacht, der mir ausführlich darlegte, warum er - trotz betonter Gegnerschaft der Entente u. pazifist. Ideen gegenüber - seine eigene Teilnahme an führender Stelle für richtig halte und daß die Jüngeren hier die alte Garde nicht im Stich lassen sollten. Immerhin: Ihr Hauptargument: ,wir sind in 1. Linie Menschen, nur in 2. Mathematiker mache ich mir völlig zu eigen, es ist auch meine oft gebrauchte Devise. ${ }^{6}$ 
Es mag dahingestellt bleiben, ob Fraenkel hier aus taktischen Gründen Hilberts Aussagen etwas entstellt hat, oder ob Hilbert selbst in seiner Argumentation wirklich Zugeständnisse an die dominierenden Anti-Versailles Stimmungen unter den deutschen Professoren und Studenten gemacht hatte, was den Hilbert-Kenner überraschen würde. Jedenfalls ist das indirekte Zitat von Hasse eindrucksvoll, der offenbar meinte, als „Mensch“ 1928 trotz des inzwischen abebbenden westlichen Wissenschaftsboykotts nicht nach Bologna reisen zu können, ehe Deutschland nicht international wieder volle Anerkennung erhalten würde.

Der 37-jährige Fraenkel dagegen schreibt in demselben Brief vom 14. Juni 1928 an den noch nicht 30-jährigen Hasse, dass er sich ihm „vermöge Ihrer seltenen Vereinigung von Menschen und Forschertum so verehrungsvoll zugetan fühle." Dieses uns heute sicher ungewöhnlich anmutende Bekenntnis vermittelt immerhin einen Eindruck von dem persönlichen Charme und von dem Charisma, das Hasse besessen haben muss. Man kann möglicherweise annehmen, dass Fraenkel anerkannte, dass Hasse überhaupt politisch dachte und kein mathematischer „Fachidiot" war.

Hasse, der wie Fraenkel,7 aber doch in anderer Weise, durch den Weltkrieg geprägt war, wo er sich freiwillig zur Marine gemeldet hatte, wusste, dass er in der Weimarer Republik politisch eher am rechten Rand stand. Am besten hat Hasse selbst in einem Interview mit Constance Reid seine Position beschrieben, in dem er sich geradezu politisch rechts von der NSDAP einordnete:

My political feelings have never been NationalSocialistic but rather 'national' in the sense of the Deutschnationale Partei, which succeeded the Conservative Party of the Second Empire [under Wilhelm II]. I had strong feelings for Germany as it was created by Bismarck in 1871 . When this was heavily damaged by the Treaty of Versailles in 1919, I resented that very much. I approved with all my heart and soul of Hitler's endeavors to remove the injustices done to Germany in that treaty. ${ }^{8}$

Auch aus dieser politischen und menschlichen Sicht ist die Diskussion der Nachfolge von Steinitz in Kiel 1928 zu beurteilen, zu der wir jetzt zurückkehren.

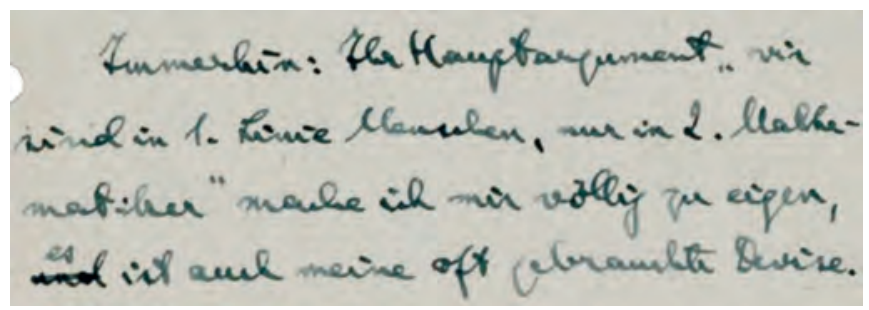

Ausschnitt aus Fraenkels Brief an Hasse, Kiel 14. 6. 28, S. 5 (Mit freundlicher Genehmigung der Göttinger Handschriftenabteilung)
Die Aufstellung der Kieler Liste für die Nachfolge von Steinitz

Fraenkel will den von ihm als Mathematiker bewunderten und mit ihm trotz politischer Meinungsunterschiede befreundeten Helmut Hasse aus Halle für die Nachfolge gewinnen. Er schreibt ihm am 8. Oktober 1928 einen siebenseitigen handschriftlichen Brief mit Vorschlägen für die Liste, woraufhin Hasse zwei Tage später ebenfalls handschriftlich mit 16 Seiten antwortet. In der von Fraenkel vorgeschlagenen und von Hasse stark korrigierten Liste steht Letzterer jeweils auf dem ersten Platz, aber es kommt den beiden sehr auf die nach Hasse zu Platzierenden an. Hasse lässt nämlich in seinem Brief keinen Zweifel daran,

daß ich die Absicht habe, Hensels Nachfolger in Marburg zu werden, eine Aussicht, die aller Voraussicht nach zum 1. April 1930 aktuell wird. 9

Er ist somit noch unentschlossen, einen etwaigen Ruf nach Kiel überhaupt anzunehmen. Es scheint Hasse in erster Linie darum zu gehen, durch die Kieler Berufung seine Position bei einer späteren Berufung nach Marburg zu verbessern, dabei zugleich die Stimmung für ihn im Kultusministerium auszuloten. Hasse wurde übrigens noch eher als erwartet, bereits im Oktober 1929, nach Marburg berufen, während die Kieler Liste vom Ministerium in Berlin auf den Kopf gestellt und der an dritter Stelle genannte Theodor Kaluza (1885-1954), Professor für angewandte Mathematik, am 27. Februar 1929 berufen wurde. ${ }^{10}$

Obwohl Fraenkel durch seine mengentheoretischen Arbeiten mittlerweile einen guten Ruf als Mathematiker hatte, war er sehr selbstkritisch und kannte seine Grenzen, wie auch aus seiner Autobiografie von 1967 hervorgeht [2]. Dies scheint auch in seinen Vorschlägen für die Nachfolge von Steinitz durch - siehe unten seine Reaktion auf Hasses Vorschlag, Max Dehn auf die Liste zu setzen. Offensichtlich wünschte er sich einen menschlich sehr angenehmen zukünftigen Kollegen, der ihn eine mögliche mathematische Überlegenheit nicht spüren lassen und insbesondere auch sein in manchen Kreisen etwas umstrittenes, abstraktes Forschungsgebiet und seine relativ geringe und einseitige Produktivität tolerieren würde.

Auch in diesem Lichte sind wohl Fraenkels Bedenken gegen eine mögliche Berufung von Emmy Noether aus Göttingen zu sehen, die unten zur Sprache kommen werden. Anders als er und Hasse, die beide erheblich jünger als Noether waren, hatte diese bisher keine feste Stelle im deutschen Hochschulsystem erhalten. Fraenkel wusste zweifellos, dass ihr Werk noch stärker als sein eigenes in der Tradition der von Steinitz begründeten abstrakten Körpertheorie stand.

Der große Hermann Weyl (1885-1955), der Nachfolger von Hilbert in Göttingen, sagte über Emmy Noether in seinem Nachruf von 1935:

I was ashamed to occupy such a preferred position beside her whom I knew to be my superior as a mathematician in many respects. [12, p. 208] 


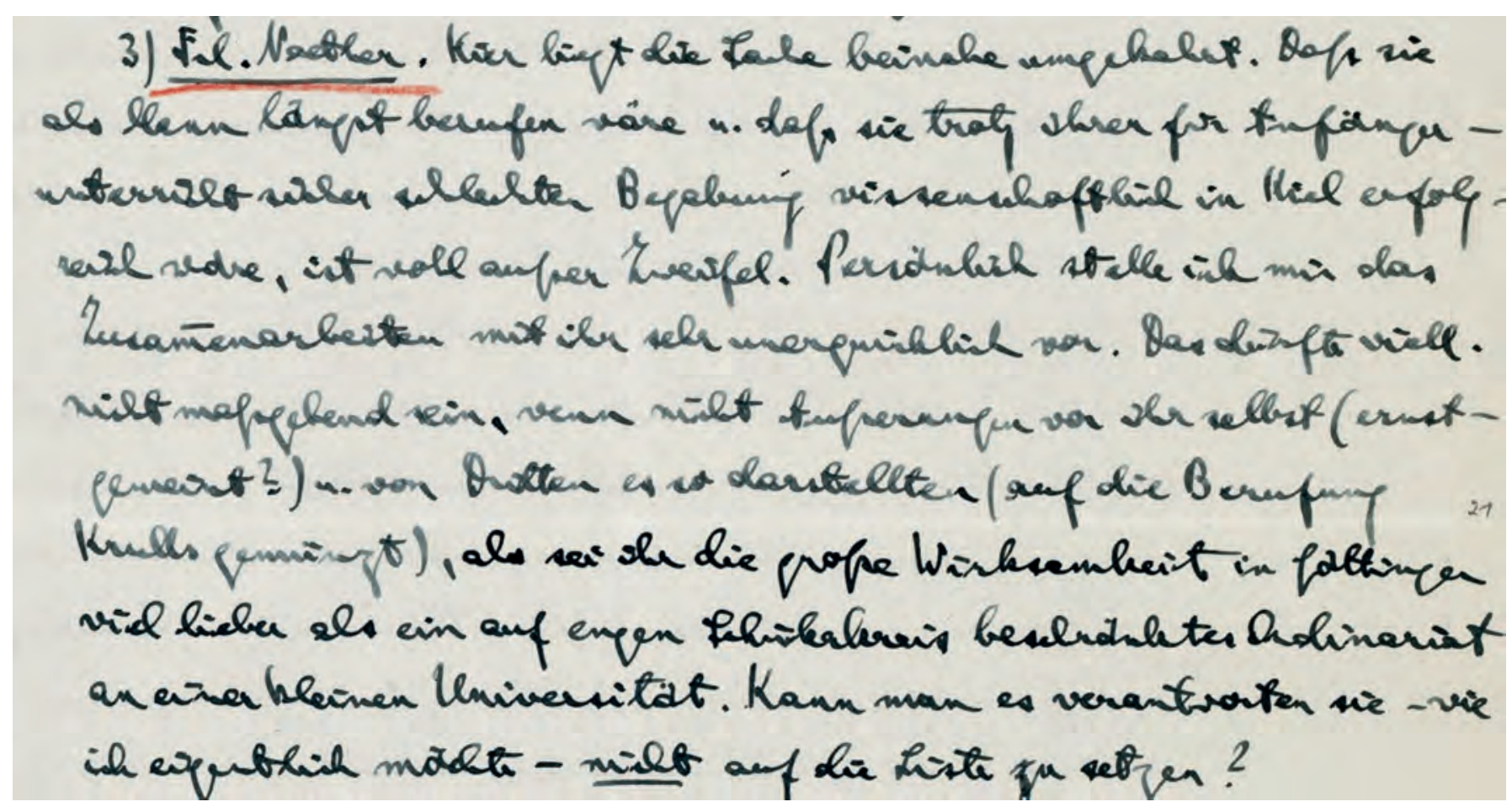

Ausschnitt aus Fraenkels Brief an Hasse, Amsterdam 8. 10. 28. (Mit freundlicher Genehmigung der Göttinger Handschriftenabteilung)

Fraenkel war um Fairness und Objektivität bemüht. In seinem handschriftlichen Brief an Hasse vom 8. Oktober 1928 aus Amsterdam, auf den vor kurzem Mechthild Koreuber in ihrem interessanten Buch [4] über Emmy Noether aufmerksam gemacht hat, sagt er etwas halbherzig das Folgende:

3) Frl. Noether. Hier liegt die Sache beinahe umgekehrt. Daß sie als Mann längst berufen wäre u. daß sie trotz ihrer für Anfängerunterricht sicher schlechten Begabung wissenschaftlich in Kiel erfolgreich wäre, ist wohl außer Zweifel. Persönlich stelle ich mir das Zusammenarbeiten mit ihr sehr unerquicklich vor. Das dürfte viell. nicht maßgebend sein, wenn nicht Äußerungen von ihr selbst (ernst gemeint?) u. von Dritten es so darstellten (auf die Berufung Krulls gemünzt), als sei ihr die große Wirksamkeit in Göttingen viel lieber als ein auf engen Schülerkreis beschränktes Ordinariat an einer kleinen Universität. Kann man es verantworten, sie - wie ich eigentlich möchte - nicht auf die Liste zu setzen? ${ }^{11}$

Fraenkels Bemerkung „Hier liegt die Sache beinahe umgekehrt" bezieht sich auf seine vorherige Nennung des Logikers Paul Bernays (1888-1977), dessen Berufung er für ausgeschlossen hält, obwohl ihm bisher „Unrecht geschehen“ sei, da „er und ich zusammen eine viel zu einseitige Vertretung der Math. in Kiel darstellen würden."

Man kann also davon ausgehen, dass Fraenkel bei einer möglichen Berufung Noethers nicht das Fehlen eines Analytikers in Kiel, sondern die unzureichende Besetzung der Anfängervorlesungen fürchtete. Das Pädagogische spielte bei Fraenkel stets eine große Rolle [2]. Zudem scheint Fraenkel sein eigenes mögliches Verhältnis zu Noether wegen ihrer besonderen genialischen Persönlichkeit beunruhigt zu haben. Dagegen findet man keine explizite Frauendiskriminierung in Fraenkels Brief, vielmehr im Gegenteil das Eingeständnis „daß sie als Mann längst berufen wäre“, was ihn offenbar zu ihrer Nennung aus schlechtem Gewissen veranlasste.

Aber Fraenkel wurde das Gewissen schnell erleichtert durch Hasses Reaktion zwei Tage später. Erneut an Hasse schreibend sagte Fraenkel daraufhin in einem Brief vom 14. Oktober 1928, immer noch aus Amsterdam:

Zu Frl. Noether ermutigen mich Ihre Bemerkungen, denen ich mindestens, was große Univers. betrifft, zustimme, guten Gewissens von ihrer Nennung abzusehen. ${ }^{12}$

Koreuber sagt an dieser Stelle, dass sie über die ermutigende Bemerkung Hasses an dieser Stelle nur spekulieren könne, da sein Brief nicht zur Verfügung stehe. Ihrer Erfahrung als Frauenbeauftragte der Freien Universität in Berlin kommt Gewicht zu, wenn sie sagt:

Analysen heutiger Berufungsverfahren zeigen, dass gerne etwas über die Wissenschaftlerinnen angenommen wird, das zur Ablehnung führen kann und führt, ohne es durch Rückfrage zu verifizieren. [4, S. 53]

Jedoch trifft diese Bemerkung eher als Kommentar auf Fraenkels Brief zu, und der Brief Hasses vom 1o. Oktober steht, wie bereits erwähnt, zur Verfügung und befindet sich 


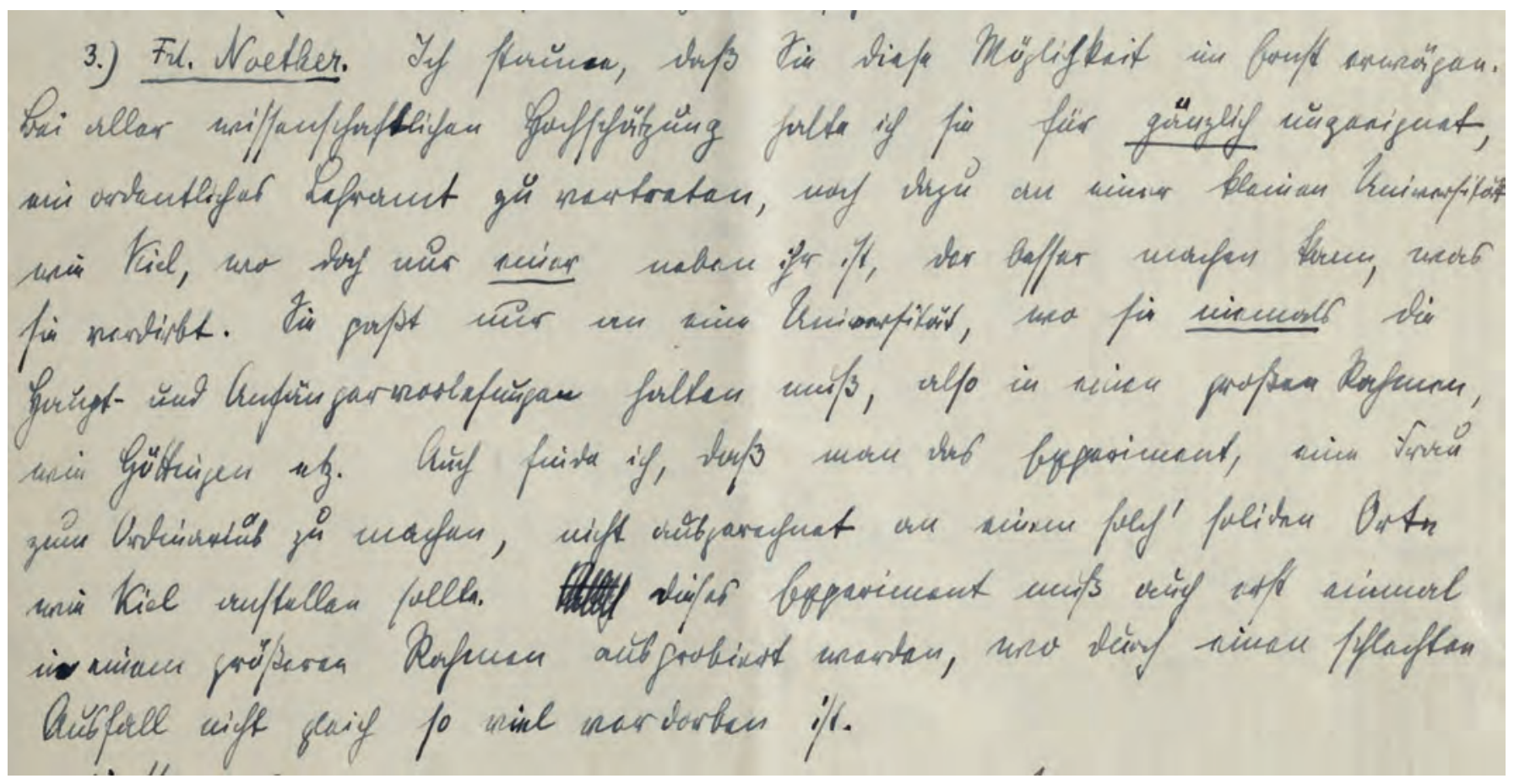

Ausschnitt aus Hasses Brief an Fraenkel, Halle 10.10.28 (Mit freundlicher Genehmigung der National Library of Israel, Jerusalem, Fraenkel Archives, File correspondence Hasse-Fraenkel)

in Fraenkels Nachlass in der Nationalbibliothek in Jerusalem. Dort liest man die folgenden Meinungen, die vermutlich von nicht wenigen Kollegen Hasses in der Weimarer Republik geteilt wurden, die aber dennoch schockieren, zumal Hasse eng mit Noether zusammenarbeitete:

3.) Frl. Noether. Ich staune, daß Sie diese Möglichkeit im Ernst erwägen. Bei aller wissenschaftlichen Hochschätzung halte ich sie für gänzlich ungeeignet, ein ordentliches Lehramt zu vertreten, noch dazu an einer kleinen Universität wie Kiel, wo doch nur einer neben ihr ist, der besser machen kann, was sie verdirbt. Sie paßt nur an eine Universität, wo sie niemals die Haupt- und Anfängervorlesungen halten muß, also in einen großen Rahmen, wie Göttingen etc. Auch finde ich, daß man das Experiment, eine Frau zum Ordinarius zu machen, nicht ausgerechnet an einem solch' soliden Orte wie Kiel anstellen sollte. Dieses Experiment muß auch erst einmal in einem größeren Rahmen ausprobiert werden, wo durch einen schlechten Ausfall nicht gleich so viel verdorben ist. ${ }^{13}$

Hasses Bemerkungen enthalten kein antisemitisches Ressentiment, wie es auch in einem Brief an den orthodoxen Juden Fraenkel kaum denkbar wäre. ${ }^{14}$ Allerdings geht er in seinen Befürchtungen wegen der mangelnden Eignung Noethers für Anfängervorlesungen einen entscheidenden Schritt weiter als Fraenkel und dehnt die Vorbehalte auf Frauen insgesamt aus, wenn er das „Experiment, eine Frau zum Ordinarius zu machen“ lieber für größere Universitä- ten reservieren möchte. Fraenkels Zustimmung „mindestens, was große Univers. betrifft" sollte wohl auch eher als Bestätigung des Problems der Anfängervorlesungen gelesen werden und das Wort "mindestens" vielleicht sogar als Einwand gegen Hasses generell frauenfeindliche Position.

Jedenfalls findet man weder bei Fraenkel noch bei Hasse die Erkenntnis, dass Emmy Noether mit ihrem ganz anderen, vielfach erschwerten akademischen Bildungsweg, mit ihrer lange verhinderten Habilitation [4, 19 ff.], kaum Möglichkeiten gehabt hatte, eine systematische Lehrpraxis zu entwickeln. ${ }^{15}$ In einem späteren Brief an Fraenkel vom 6. November unterstreicht Hasse übrigens seine Aufgeschlossenheit gegenüber jüdischen männlichen Kollegen, wenn er die Verwandtschaft der Geistesrichtungen von Steinitz und des mit diesem "gut befreundeten" namhaften Topologen Max Dehn (1878-1952) betont und damit Dehn erstmals in die Diskussion für die Kieler Liste bringt: „Als Nachfolger von Steinitz scheint mir z. B. auch ein Mann wie M. Dehn in hervorragender Weise geeignet. " ${ }^{16}$

Fraenkels Reaktion darauf scheint anzudeuten, dass er die manchmal scharfe polemische Art Dehns fürchtete, wiederum vielleicht Ausdruck seines Unwohlseins mit zu ungezügelter Wettbewerbsorientierung, die ihm der aus der Göttinger Schule stammende Spitzenmathematiker Dehn zu verkörpern schien. Fraenkel schreibt am 15. November 1928 an Hasse:

Dehn, den Sie vorschlugen, kommt (auch abges. vom Alter und von der hies. Abneigung gegen etwa gar 2 Juden auf d. Liste) m. E. gar nicht in Betracht; erstens, weil weder das Minist. einen Fkfrter [Frankfurter] 
Ordinarius beruft noch er selbst wohl gekommen wäre, zweitens, weil mir seine etwas überhebliche Art der Klassifizierung nicht nur der Math'er, sondern auch der math. Forschungsrichtungen gar nicht als angenehme Nachbarschaft erschienen wäre. ${ }^{17}$

Auf derselben Seite dieses Briefes äußert Fraenkel übrigens auch gewisse Minderwertigkeitskomplexe gegenüber Hasse bei etwaiger Zusammenarbeit mit ihm. Er sagt: „Meine Forschungsfähigkeiten sind nicht meine stärkste Seite“ und er beklagt das „Nachlassen meines Gedächtnisses“. Im selben Brief berichtet Fraenkel Hasse von der Fertigstellung der Kieler Berufungsliste und sagt: „In einer Präambel, die viel Diskussion verursacht hat [...] sind Bernays und E. Noether ausdrücklich genannt, aber als für hier ungeeignet erklärt." In der offiziellen Fakultätsliste, die im Geheimen Staatsarchiv in Berlin aufbewahrt wird, heißt es tatsächlich:

Sie [die Fakultät] muss hierbei von vorneherein absehen von der Nennung zweier Persönlichkeiten, an die zu denken in diesem Falle nahe läge und die der Bekleidung eines Ordinariats längst würdig sind, der n. b. a.o. [nichtbeamteten außerordentlichen] Professoren an der Universität Göttingen Herrn Dr. P. Bernays und Frl. Dr. E. Noether. Beide kommen nach einstimmiger Meinung der Fakultät für die hiesigen Verhältnisse keinesfalls in Frage; jener, weil sich seine Forschungsrichtung mit der des anderen hiesigen Fachvertreters allzu stark berührt, diese, weil ihre ausschliessliche Einstellung auf besonders begabte Studierende sich an einer kleineren Universität wie der unsrigen zu einer Gefährdung des Lehrbetriebs auswirken müsste. ${ }^{18}$

Man darf wohl annehmen, auch im Lichte der letzten Bemerkung Fraenkels über Dehn, dass jene „Diskussion“ in der Fakultät eher antisemitisch und gegen Bernays und Noether als „würdige“ Kandidaten gerichtet war, dass sie also eher eine vollständige Unterdrückung der beiden Namen als deren Berücksichtigung in der regulären Liste zum Gegenstand hatte. In dieser Beziehung war wohl Fraenkel eher noch ein progressiver Außenseiter unter den deutschen Professoren, und er hat wenigstens für die Nennung der Namen der beiden prominenten Mathematiker in der Präambel gesorgt, die beide - ebenso wie Fraenkel selbst - fünf Jahre später von den Nazis aus Deutschland vertrieben wurden und im Unterschied zu Fraenkel nie eine angemessene akademische Position erreichen sollten.

\section{Schlussbemerkungen}

Es ist wahrscheinlich, dass in der Weimarer Republik andere Berufungskommissionen als die Kieler das „NoetherProblem“ auf ähnliche Weise „gelöst“ haben. Es ist nicht zu bezweifeln, dass Bedenken wegen vermeintlicher mangelnder Eignung von Emmy Noether für Anfängervorlesungen weit verbreitet und teilweise berechtigt waren. Unser Beispiel hat aber gezeigt oder wenigstens angedeutet, dass antiemanzipatorische (Hasse) und antisemitische (im Falle der
Kieler Fakultät) Motive eine Rolle im Hintergrund spielen konnten.

Man weiß auch um die traditionelle Dominanz der Forschungskompetenz im akademischen Beförderungssystem, und man muss deshalb die Hervorhebung des Pädagogischen hier als problematisch empfinden. Fraenkel selbst schrieb ja an Hasse klar und deutlich über Noether, dass „sie als Mann längst berufen wäre $u$. daß sie trotz ihrer für Anfängerunterricht sicher schlechten Begabung wissenschaftlich in Kiel erfolgreich wäre." Dass zudem aktive Haltungen zugunsten einer rechtzeitigen Einbeziehung von Frauen in die Lehre unter den deutschen Universitätsprofessoren rar waren, ist durch viele Erfahrungen von Akademikerinnen der damaligen Zeit verbürgt [11].

Fast möchte man - auf Fraenkel und Hasse bezogen und ihr Credo etwas überspitzt paraphrasierend - sagen, dass sie in erster Linie Männer, nur in zweiter Linie Mathematiker waren.

Anmerkungen

1. Dieses Archivmaterial stammt aus der Niedersächsischen Staatsund Universitätsbibliothek Göttingen, Abteilung für Handschriften und Seltene Drucke (im Folgenden zitiert als Göttinger Handschriftenabteilung) (Korrespondenz Hasse), aus dem Geheimen Staatsarchiv Preußischer Kulturbesitz, Berlin (zitiert als GStA PK), wo sich die Akten des damaligen Preußischen Kultusministeriums betreffend die Universität Kiel befinden; ferner aus dem Hessischen Staatsarchiv Marburg, wo die Marburger Universitätsakten liegen, und schließlich aus The National Library of Israel, Jerusalem, wo sich die Fraenkel Archives befinden. Allen Archiven wird für die Vermittlung der Materialien und für die Erlaubnis, Faksimiles daraus zu publizieren, gedankt.

2. Hier ist besonders an die große algebrentheoretische Arbeit gemeinsam mit Richard Brauer [1] und an die Korrespondenz zwischen Hasse und Noether [5] zu denken. Die Korrespondenz ist vorwiegend mathematisch und geht kaum auf Berufungsfragen ein, eher auf die wünschenswerte Förderung für Noethers Schüler.

3. Fraenkels interessante Autobiografie ist vor Kurzem erstmals in englischer Übersetzung erschienen [2]. Leider sind die teilweise auf Erinnerungsfehlern Fraenkels beruhenden Irrtümer nicht beseitigt oder kommentiert worden, da die Herausgeberin kaum mathematikhistorische Literatur und auch nicht Fraenkels Korrespondenz verwendet hat. Siehe meine Rezension in Historia Mathematica 44 (2017), 288-292.

4. Fraenkel an Hasse, Kiel 13.12.31, Handschrift. Göttinger Handschriftenabteilung, cod. H. Hasse, 32-2, Blatt 421.

5. GStA, Rep. 76Va, Sekt. 9, Tit. 4, no. 22, „Durchführung des BBG an der Universität Kiel“, B1. 57.

6. Fraenkel an Hasse, Kiel 14. 6. 28. Handschrift. Göttinger Handschriftenabteilung, cod. H. Hasse, 1- 474, Brief no. 7, S. 4/5. Vgl. Faksimile Fraenkel. Zur Bologna-Affäre und zu Hilberts Standpunkt zur internationalen Zusammenarbeit vgl. meinen kürzlich erschienenen Artikel [10]. Die Tatsache, dass der Kongressteilnehmer Fraenkel in seiner Korrespondenz nichts von einer politischen Rede Hilberts in Bologna schreibt, ist für mich ein weiterer indirekter Beweis dafür, dass Hilbert eine solche Rede dort nicht gehalten hat.

7. Fraenkel hatte sich während des Krieges freiwillig als jüdischer Feldkaplan einsetzen lassen, betonte aber in dem oben zitierten NaziFragebogen von 1933, „aus meiner Pflichterfüllung im Feld keine Vorteile für mich herleiten zu wollen“. Ebd. Anmerkung 5, Bl. 55. Dies war wohl als eine Art von Protest zu verstehen gegen die willkürlichen Entlassungen jüdischer Beamter, von denen die Nazis für den Zeitraum von zwei Jahren sogenannte „Frontkämpfer“ ausnahmen.

8. [6, p. 250], auch zitiert in [7, p. 47].

9. Hasse an Fraenkel, Halle, 10. Oktober 1928. The Jewish National and University Library, Jerusalem Abraham Fraenkel Archive.

1o. GStA PK, I. HA Rep. 76 Kultusministerium, Va Sekt. 9 Tit. IV Nr. 1 Bd. 21, Blatt 392-393. Bereits am 12. Februar 1929 war Hensel in Marburg mit Wirkung Ende März emeritiert worden, anscheinend ein Jahr vorzeitig, möglicherweise auf Grund der Notverordnungen. Hessi- 
sches Staatsarchiv Marburg, Best 310 Kuratorium Acc 195176, No. 319. Man muss wohl davon ausgehen, dass die Nichtberufung von Hasse in Kiel mit der bevorstehenden Emeritierung von Hensel in Marburg im Zusammenhang stand, da beide Universitäten dem preußischen Kultusministerium unterstanden.

11. [4, S. 52/53]. Ich habe einige Lesefehler nach dem Original in der Göttinger Handschriftenabteilung korrigiert, von denen aber nur das Wort „unerträglich“ (anstatt richtig „unerquicklich“) sinnentstellend ist. S. Faksimile Fraenkel.

12. [4, S. 53], wo der Brief versehentlich mit 10.10. 28 datiert wird. Original in der Göttinger Handschriftenabteilung, cod. H. Hasse, 1-474, Brief no. 9, Seite 3.

13. The National Library of Israel, Jerusalem, Fraenkel archives, file correspondence Hasse-Fraenkel, Hasse an Fraenkel, Halle 10. Oktober 28, Original Hand, 16 Seiten, Seite 8.

14. Die DNVP, der Hasse damals politisch nahestand, hatte aber starke Tendenzen zum Antisemitismus. Unter den Bedingungen der NaziDiktatur schließlich schockte Hasse amerikanische Kollegen damit, dass er von einem „war between the Germans and the Jews" sprach und damit die antisemitischen Maßnahmen in der Redaktion des Zentralblatts (1938) verteidigte. S. [8, S. 164].

15. Man erinnert sich auch daran, dass etwa zur selben Zeit in Berlin (1927) das Habilitationsverfahren der angewandten Mathematikerin Hilda Geiringer mit ungefähr dem entgegengesetzten Argument, unzureichender mathematischer Strenge (übrigens nicht mangelnder Produktivität!), von den reinen Mathematikern in Frage gestellt wurde, während unzureichende Produktivität des ebenfalls zur Habilitation anstehenden Georg Feigl viel milder beurteilt wurde. Die traditionsbewussten Männer wählten also ihre Argumente taktisch je nach Bedarf. Vgl. [9].

16. Quelle wie in Anmerkung 13. Hasse an Fraenkel, Halle 6. November 1928, Original Hand, 3 Seiten, Seite 2.

17. Fraenkel an Hasse, Kiel 15.11.28. Handschrift. Göttinger Handschriftenabteilung, cod. H. Hasse, 1-474, Brief no. 12. Bl. $12 / 2$.

18. GStA PK, I. HA Rep. 76 Kultusministerium, Va Sekt. 9 Tit. IV Nr. 1 Bd. 21, Blatt 38o. Kiel, Philosophische Fakultät, 15 . November 1928 (maschineschriftlich).

\section{Literatur}

[1] Brauer, R., H. Hasse, und E. Noether (1932), Beweis eines Hauptsatzes in der Theorie der Algebren. Journal für die reine und angewandte Mathematik 167, 399-404.

[2] Fraenkel, A. A. (2016), Recollections of a Jewish Mathematician in Germany. Edited by Jiska Cohen-Mansfield, Switzerland, Birkhäuser. (ursprünglich erschienen auf Deutsch als Lebenskreise in Stuttgart, DVA, 1967).

[3] Hasse, H. (1933), Die Struktur der R. Brauerschen Algebrenklassengruppe über einem algebraischen Zahlkörper ... Mathematische Annalen 107, 731-760.

[4] Koreuber, M. (2015), Emmy Noether, die Noether-Schule und die Moderne Algebra. Zur Geschichte einer kulturellen Bewegung. Berlin, Springer.

[5] Lemmermeyer, F. und P. Roquette (Hrg., 2006): Helmut Hasse und Emmy Noether. Die Korrespondenz 1925-1935. Göttingen, Universitätsverlag.

[6] Reid, C. (1976), Courant in Göttingen and New York. The Story of an Improbable Mathematician. Springer, New York.

[7] Segal, S. (1980), Helmut Hasse in 1934. Historia Mathematica 7, $46-56$.

[8] Siegmund-Schultze, R. (1993a), Mathematische Berichterstattung in Hitlerdeutschland. Der Niedergang des Jahrbuchs über die Fortschritte der Mathematik (1869-1945). Göttingen, Vandenhoeck \& Ruprecht.

[9] Siegmund-Schultze, R. (1993b), Hilda Geiringer-von Mises, Charlier Series, Ideology, and the Human Side of the Emancipation of Applied Mathematics at the University of Berlin during the 1920s. Historia Mathematica 20, 364-381.

[10] Siegmund-Schultze, R. (2016), "Mathematics Knows No Races": A Political Speech that David Hilbert Planned for the ICM in Bologna in 1928. The Mathematical Intelligencer 38, no. 1, 56-66.

[11] Tobies, R. (Hrg. 2008), Aller Männerkultur zum Trotz. Frauen in Mathematik, Naturwissenschaften und Technik. Frankfurt, Campus.

[12] Weyl, H. (1935), Emmy Noether. Scripta Mathematica 3, 201-220.

Prof. Dr. Reinhard Siegmund-Schultze, University of Agder, Faculty of Engineering and Science,

Gimlemoen 25a, Postboks 422, 4604 Kristiansand S, Norwegen, reinhard.siegmund-schultze@uia.no

Reinhard Siegmund-Schultze ist 1953 in Halle geboren, wo er Mathematik studiert und 1979 zur Geschichte der

Funktionalanalysis promoviert hat. Im Jahre 2000 erhielt er eine Stelle als Mathematikhistoriker in Kristiansand

(Norwegen). Er schreibt gegenwärtig an einer Biografie des angewandten Mathematikers und Ingenieurs Richard von Mises (1883-1953). 